\title{
Facile $a b$ initio approach for self-localized polarons from canonical transformations
}

\author{
Nien-En Lee, ${ }^{1,2}$ Hsiao-Yi Chen $\odot,{ }^{1,2}$ Jin-Jian Zhou, ${ }^{1}$ and Marco Bernardi ${ }^{1}$ \\ ${ }^{1}$ Department of Applied Physics and Materials Science, California Institute of Technology, Pasadena, California 91125, USA \\ ${ }^{2}$ Department of Physics, California Institute of Technology, Pasadena, California 91125, USA
}

(Received 9 November 2020; revised 24 April 2021; accepted 8 June 2021; published 24 June 2021)

\begin{abstract}
Electronic states in a crystal can localize due to strong electron-phonon (e-ph) interactions, forming so-called small polarons. Methods to predict the formation and energetics of small polarons are either computationally costly or not geared toward quantitative predictions. Here we show a formalism based on canonical transformations to compute the polaron formation energy and wave function using ab initio $e$-ph interactions. Comparison of the calculated polaron and band-edge energies allows us to determine whether charge carriers in a material favor a localized small polaron over a delocalized Bloch state. Due to its low computational cost, our approach enables efficient studies of the formation and energetics of small polarons, as we demonstrate by investigating electron and hole polaron formation in alkali halides and metal oxides and peroxides. We outline refinements of our scheme and extensions to compute transport in the polaron hopping regime.
\end{abstract}

DOI: 10.1103/PhysRevMaterials.5.063805

\section{INTRODUCTION}

Self-localized (small) polarons are charge carriers that interact strongly with the lattice vibrations, becoming trapped as a result of the local lattice distortion [1]. Small polarons are essential to understanding electrical transport and optical properties in a wide range of materials, including transitionmetal oxides, alkali halides, and organic molecular crystals [2-5]. The presence of small polarons in these materials is typically associated with a diffusive, thermally activated charge-transport regime characterized by low mobility values, typically less than $1 \mathrm{~cm}^{2} / \mathrm{V} \mathrm{s}$ [6]. Recent progress has enabled direct observation of small polaron states [7,8] and clarified their important role in various technologies [9-13].

The theoretical treatment of small polarons was pioneered by Holstein [14] based on ideas from Landau and Pekar [15]. It was later extended by Lang and Firsov [16] and generalized by Munn and Silbey [17,18] and Hannewald et al. [19] to improve the description of electron-phonon ( $e$-ph) interactions. The resulting small polaron theory can qualitatively demonstrate the transition from bandlike to hopping transport observed in experiments [4,20,21]. Yet, the presence of a selflocalized polaron state is typically assumed in these theories rather than directly predicted, and most theoretical treatments of polarons are not geared toward quantitative predictions on real materials as they rely on empirical parameters and take into account only one or a few vibrational modes.

Early work formulated the problem of polaron formation as a competition of energies for localizing an electronic state, which relaxes the lattice but increases the electron kinetic energy [22]. Despite this intuition, whether charge carriers form small polarons or not remains controversial in many materials. For example, photoemission experiments found no evidence of small polarons in $\mathrm{SrTiO}_{3}$ [23], although mobility and optical measurements suggested their presence $[24,25]$.

First-principles calculations can accurately compute the electronic structure, lattice dynamics, and $e$-ph coupling [26], and they are ideally suited to provide quantitative approaches for treating both large and small polarons. However, existing studies have focused on semiconductors and insulators without small polaron effects [27-33]. First-principles calculations of small polarons involve supercells with excess charge or defects explicitly added [34-37]. While useful, these approaches require computationally costly calculations with many atoms, and their reliability is limited by the accuracy of density functional theory (DFT) exchange-correlation functionals and the treatment of charged systems in DFT. A rigorous and convenient first-principles approach connecting standard small polaron theory [38] and modern $a b$ initio $e$-ph calculations would be expedient.

Here we show an efficient approach to compute the small polaron energy in a localized basis starting from a trial polaron wave function. Employing a canonical transformation formalism [14], we construct a self-localized polaron state that is free from hopping and decoupled from all vibrational modes [39]. We determine whether an electron or hole charge carrier self-localizes by comparing the energy of the polaron state with the conduction- or valence-band edge, thus predicting whether a small polaron forms and determining its formation energy. The computational cost of our scheme is equivalent to a DFT calculation on a unit cell plus an inexpensive $e$-ph computational step. Its efficiency allows us to investigate small polarons in various alkali halides, oxides, and perovskites with minimal computational effort. Our work bridges the gap between standard small polaron theory and modern $a b$ initio $e$-ph calculations.

\section{THEORY}

\section{A. Polaron Hamiltonian}

We derive the effective small polaron Hamiltonian in a distorted lattice through a canonical transformation [14], inspired by the treatment of the charged harmonic oscillator (CHO) 
in an external electric field $E$ [40]. The Hamiltonian of a one-dimensional $\mathrm{CHO}$ is

$$
H^{(\mathrm{CHO})}=\omega\left(b^{\dagger} b+\frac{1}{2}\right)+\omega g\left(b+b^{\dagger}\right),
$$

where $b^{\dagger}$ and $b$ are oscillator creation and annihilation operators, and the coupling parameter is $g=e E / \sqrt{2 m \omega^{3}}$, with $e$, $m$, and $\omega$ the charge, mass, and frequency of the oscillator, respectively. Here and below we set $\hbar=1$. To solve the CHO Hamiltonian, the common approach is to stretch the oscillator spring to its new equilibrium position using the canonical transformation of operators $O \rightarrow \widetilde{O}=e^{S} O e^{-S}$. Defining the CHO generator as $S^{(\mathrm{CHO})}=g\left(b^{\dagger}-b\right)$, this transformation gives

$$
\begin{gathered}
\tilde{b}=b-g, \\
\widetilde{H}^{\text {(CHO) }}=\omega\left(b^{\dagger} b+\frac{1}{2}\right)-\omega g^{2} .
\end{gathered}
$$

The shift of the operator $b$ in Eq. (2) amounts to shifting the coordinate system:

$$
\widetilde{x}=\frac{1}{\sqrt{2 m \omega}}\left(\tilde{b}^{\dagger}+\widetilde{b}\right)=\frac{1}{\sqrt{2 m \omega}}\left(b^{\dagger}+b\right)+x_{0},
$$

where $x$ is the position operator and $x_{0}=-2 g / \sqrt{2 m \omega}$ is the new equilibrium position. The second term in Eq. (3) is always negative and can be interpreted as the energy decrease resulting from relaxing the oscillator to a new equilibrium position due to the electrical force, because $-\omega g^{2}=\frac{1}{2} m \omega^{2} x_{0}^{2}+e E x_{0}$.

Inspired by Holstein's treatment [14], we perform an analogous transformation on the $e$-ph Hamiltonian in the electronic Wannier [41] and phonon momentum basis,

$$
\begin{aligned}
H= & \sum_{m n} \varepsilon_{m n} a_{m}^{\dagger} a_{n}+\sum_{\mathbf{Q}} \omega_{\mathbf{Q}}\left(b_{\mathbf{Q}}^{\dagger} b_{\mathbf{Q}}+\frac{1}{2}\right) \\
& +\frac{1}{\sqrt{N_{\Omega}}} \sum_{m n} \sum_{\mathbf{Q}} \omega_{\mathbf{Q}} g_{\mathbf{Q} m n}\left(b_{\mathbf{Q}}^{\dagger}+b_{-\mathbf{Q}}\right) a_{m}^{\dagger} a_{n} .
\end{aligned}
$$

Here, $n=j_{n} \mathbf{R}_{n}$ is a collective index labeling the $j_{n}$ th Wannier function (WF) in the unit cell with the origin at the Bravais lattice vector $\mathbf{R}_{n}$, while $a_{n}=a_{j_{n} \mathbf{R}_{n}}$ is the corresponding electron annihilation operator and $b_{\mathbf{Q}}$ is the phonon annihilation operator, where $\mathbf{Q}$ is a collective label for the phonon mode $v$ and momentum q. The hopping strength and phonon energy are denoted as $\varepsilon_{m n}$ and $\omega_{\mathbf{Q}}$, respectively, and $N_{\Omega}$ is the number of unit cells in the crystal. The unitless $e$-ph coupling matrix element $g_{\mathbf{Q} m n}$ is obtained by transforming to the electron Wannier basis a unitless $e$-ph matrix element in momentum space, $\tilde{g}_{m n v}(\mathbf{k}, \mathbf{q})=g_{m n v}(\mathbf{k}, \mathbf{q}) /\left(\hbar \omega_{\nu \mathbf{q}}\right)$, where $g_{m n v}(\mathbf{k}, \mathbf{q})$ is defined in Eq. (24) of Ref. [42]. Using the notation in Ref. [42], the explicit definition is $g_{\mathbf{Q} m n} \equiv \tilde{g}_{m n v}\left(\mathbf{R}_{e}, \mathbf{q}\right)=$ $\frac{1}{N_{e}} \sum_{\mathbf{k}, i j} e^{-i \mathbf{k} \cdot \mathbf{R}_{e}} \mathcal{U}_{m i}^{\dagger}(\mathbf{k}+\mathbf{q}) \tilde{g}_{i j v}(\mathbf{k}, \mathbf{q}) \mathcal{U}_{j n}(\mathbf{k})$, where $\mathcal{U}$ are unitary Wannier gauge matrices [42].

We define the generator $S$ as

$$
\begin{gathered}
S=\sum_{m n} C_{m n} a_{m}^{\dagger} a_{n}, \\
C_{m n}=\frac{1}{\sqrt{N_{\Omega}}} \sum_{\mathbf{Q}} B_{\mathbf{Q} m n}\left(b_{\mathbf{Q}}^{\dagger}-b_{-\mathbf{Q}}\right),
\end{gathered}
$$

and using the transformation $O \rightarrow \widetilde{O}=e^{S} O e^{-S}$ we obtain the transformed electron and phonon annihilation operators, respectively, as

$$
\begin{gathered}
\tilde{a}_{m}=\sum_{n} e_{m n}^{-C} a_{n}, \\
\widetilde{b}_{\mathbf{Q}}=b_{\mathbf{Q}}-\frac{1}{\sqrt{N_{\Omega}}} \sum_{m n} B_{\mathbf{Q} m n} a_{m}^{\dagger} a_{n},
\end{gathered}
$$

where $e_{m n}^{-C}$ is shorthand for the phonon operator,

$$
e_{m n}^{-C}=\delta_{m n}-C_{m n}+\frac{1}{2 !} \sum_{i} C_{m i} C_{i n}-\cdots,
$$

with $C_{m n}$ defined in Eq. (7). Above, we introduced the undetermined distortion coefficients $B_{\mathbf{Q} m n}$, which, analogous to the coupling $g$ in the $\mathrm{CHO}$ example, quantify how the transformation stretches the spring of each phonon mode to a new equilibrium position due to the electrical forces applied on the lattice by the charge carrier. This physical interpretation is manifest in Eq. (9), where one changes the basis to a distorted lattice configuration in analogy with Eq. (2), implying that the operators $a_{n}^{\dagger}$ and $b_{Q}^{\dagger}$ create a polaron or phonon, respectively, in the distorted lattice. To make the transformation unitary, the distortion coefficients need to satisfy $B_{\mathbf{Q} m n}^{*}=B_{-\mathbf{Q} n m}$, so that the operators $C_{m n}$ and $S$ are both anti-Hermitian.

The polaron Hamiltonian is obtained by substituting the transformed electron and phonon operators [43]:

$$
\begin{aligned}
\widetilde{H}= & \sum_{m n} E_{m n} a_{m}^{\dagger} a_{n}+\sum_{\mathbf{Q}} \omega_{\mathbf{Q}}\left(b_{\mathbf{Q}}^{\dagger} b_{\mathbf{Q}}+\frac{1}{2}\right) \\
& +\frac{1}{\sqrt{N_{\Omega}}} \sum_{m n \mathbf{Q}} \omega_{\mathbf{Q}} G_{\mathbf{Q} m n}\left(b_{\mathbf{Q}}^{\dagger}+b_{-\mathbf{Q}}\right) a_{m}^{\dagger} a_{n},
\end{aligned}
$$

where the polaron hopping strength $E_{m n}$ and the residual polaron-phonon (pl-ph) coupling constant $G_{\mathbf{Q} m n}$ are defined, respectively, as

$$
\begin{gathered}
E_{m n}=\langle\widetilde{\varepsilon}\rangle_{m n}+\frac{1}{N_{\Omega}} \sum_{i \mathbf{Q}} \omega_{\mathbf{Q}} B_{-\mathbf{Q} m i}\left(B_{\mathbf{Q} i n}-2\left\langle\widetilde{g}_{\mathbf{Q}}\right\rangle_{i n}\right), \\
G_{\mathbf{Q} m n}=\left\langle\widetilde{g}_{\mathbf{Q}}\right\rangle_{m n}-B_{\mathbf{Q} m n},
\end{gathered}
$$

and the angle brackets $\langle\cdots\rangle$ indicate a thermal average over phonon states.

\section{B. Thermal average}

In the effective polaron Hamiltonian derived above, the transformed hopping and $e$-ph coupling matrices $\widetilde{\varepsilon}_{m n}$ and $\widetilde{g}_{\mathbf{Q} m n}$, denoted as $\widetilde{M}_{m n}$, are defined as

$$
\tilde{M}_{m n}=\sum_{i j} e_{m i}^{C} M_{i j} e_{j n}^{-C} .
$$

These transformed matrices still contain phonon operators (through the operator $C_{m n}$ ). Following Holstein [14], we take their thermal average in Eq. (12) to obtain the effective polaron Hamiltonian in Eq. (11). The thermal average is com- 
puted using the Baker-Campbell-Hausdorff formula [19]:

$$
\langle\tilde{M}\rangle_{m n}=\left\langle e^{C} M e^{-C}\right\rangle_{m n}=\left\langle M+\frac{1}{2 !}[C,[C, M]]+\cdots\right\rangle_{m n},
$$

where terms with an odd number of $C_{m n}$ vanish because the thermal average of an odd number of $b_{\mathbf{Q}}$ or $b_{\mathbf{Q}}^{\dagger}$ is zero. Substituting $C_{m n}$ as defined in Eq. (7), we get

$$
\begin{aligned}
\langle\tilde{M}\rangle_{m n}= & \left\langle\sum_{i} \frac{1}{(2 i) !}[C,[\cdots[C,[C, M]] \cdots]\rangle_{m n}\right. \\
= & \sum_{i} \frac{1}{(2 i) ! N_{\Omega}^{i}}\left[\sum_{\mathbf{Q}_{1}} B_{\mathbf{Q}_{1}},\left[\cdots\left[\sum_{\mathbf{Q}_{2 i}} B_{\mathbf{Q}_{2 i}}, M\right] \cdots\right]\right] \\
& \times\left\langle\left(b_{\mathbf{Q}_{1}}^{\dagger}-b_{-\mathbf{Q}_{1}}\right) \cdots\left(b_{\mathbf{Q}_{2 i}}^{\dagger}-b_{-\mathbf{Q}_{2 i}}\right)\right\rangle_{m n},
\end{aligned}
$$

where the phonon operator part can be factored out in the last equality because all permutations of $\left(b_{\mathbf{Q}}^{\dagger}-b_{-\mathbf{Q}}\right)$ give the same thermal average. Next, we apply the Wick theorem and use the identities $\left\langle b_{\mathbf{Q}}^{\dagger} b_{\mathbf{Q}}\right\rangle=N_{\mathbf{Q}}$ and $\left\langle b_{\mathbf{Q}} b_{\mathbf{Q}}^{\dagger}\right\rangle=N_{\mathbf{Q}}+1$ on the thermal average part, where $N_{\mathbf{Q}}$ is the phonon thermal occupation:

$$
\begin{aligned}
& \langle\tilde{M}\rangle_{m n}=\sum_{i} \frac{(-1)^{i}}{(2 i) ! N_{\Omega}^{i}} \sum_{\mathbf{Q}_{1} \cdots \mathbf{Q}_{i}}\left(2 N_{\mathbf{Q}_{1}}+1\right) \cdots\left(2 N_{\mathbf{Q}_{i}}+1\right) \\
& \times \sum_{\text {all pairings }}\left[B_{\mathbf{Q}_{1}},\left[B_{-\mathbf{Q}_{1}},\left[\cdots\left[B_{\mathbf{Q}_{i}},\left[B_{-\mathbf{Q}_{i}}, M\right]\right] \cdots\right]\right]\right]_{m n} .
\end{aligned}
$$

This expression can be simplified by assuming that all distortion coefficients $B_{\mathbf{Q}}$ commute with each other. Under this assumption, the commutator part of every possible pairing is the same, and each of the $(2 i) ! / 2^{i} i$ ! pairings gives the same contribution. We obtain

$$
\begin{aligned}
\langle\tilde{M}\rangle_{m n}= & \sum_{i} \frac{(-1)^{i}}{N_{\Omega}^{i} i !} \sum_{\mathbf{Q}_{1} \cdots \mathbf{Q}_{i}}\left(N_{\mathbf{Q}_{1}}+\frac{1}{2}\right) \cdots\left(N_{\mathbf{Q}_{i}}+\frac{1}{2}\right) \\
& \times\left[B_{\mathbf{Q}_{1}},\left[B_{-\mathbf{Q}_{1}},\left[\cdots\left[B_{\mathbf{Q}_{i}},\left[B_{-\mathbf{Q}_{i}}, M\right]\right] \cdots\right]\right]\right]_{m n} .
\end{aligned}
$$

Defining a linear operator $\Lambda$ on $M$ as

$$
\sum_{\alpha \gamma} \Lambda_{m n, \alpha \gamma} M_{\alpha \gamma}=\frac{1}{N_{\Omega}} \sum_{\mathbf{Q}}\left(N_{\mathbf{Q}}+\frac{1}{2}\right)\left[B_{\mathbf{Q}},\left[B_{-\mathbf{Q}}, M\right]\right]_{m n},
$$

we obtain the final expression

$$
\langle\tilde{M}\rangle_{m n}=\sum_{\alpha \gamma} e_{m n, \alpha \gamma}^{-\Lambda} M_{\alpha \gamma}
$$

For the special case in which all the nonlocal distortion coefficients vanish, and using the ansatz,

$$
B_{\mathbf{Q} m n}=g_{\mathbf{Q} m n} \delta_{m n},
$$

we obtain (see below) a self-localized polaron state with negligible intersite hopping. Using this ansatz, the thermal average of the transformed matrix can be written as $\langle\widetilde{M}\rangle_{m n}=$ $\exp \left[-\lambda_{m n}\right] M_{m n}[40]$, where the exponent $\lambda_{m n}(T)$ depends on temperature $T$ and on the difference between the local $e$-ph couplings at the $m$ and $n$ WF sites,

$$
\lambda_{m n}(T)=\frac{1}{N_{\Omega}} \sum_{\mathbf{Q}}\left(N_{\mathbf{Q}}(T)+\frac{1}{2}\right)\left|g_{\mathbf{Q} m m}-g_{\mathbf{Q} n n}\right|^{2},
$$

and on the phonon thermal occupation factor $N_{\mathbf{Q}}(T)$. In this work, the quantity $\lambda_{m n}$ is computed using $a b$ initio $e$-ph coupling constants $g_{\mathbf{Q} m m}$, paying attention to converge the Brillouin zone integral in Eq. (21). The diagonal part of $\lambda_{m n}$ is identically zero, which makes $\exp \left[-\lambda_{m m}\right]=1$ for all sites $m$. The off-diagonal part of $\exp \left[-\lambda_{m n}\right]$ is orders of magnitude smaller than unity (typically of order $10^{-2}-10^{-10}$ at $300 \mathrm{~K}$ ), as we verify explicitly in our numerical calculations. Thus we have

$$
\exp \left[-\lambda_{m n}\right] \approx \delta_{m n}
$$

\section{Polaron hopping and formation energy}

Substituting Eqs. (20)-(22) into Eq. (12), we derive the central equations for the polaron hopping strength $E_{m n}$ and pl-ph coupling $G_{\mathbf{Q} m n}$ :

$$
\begin{gathered}
E_{m n}=\left(\varepsilon_{m m}-\frac{1}{N_{\Omega}} \sum_{\mathbf{Q}} \omega_{\mathbf{Q}}\left|g_{\mathbf{Q} m m}\right|^{2}\right) \delta_{m n}, \\
G_{\mathbf{Q} m n}=g_{\mathbf{Q} m n} \delta_{m n}-B_{\mathbf{Q} m n}=0 .
\end{gathered}
$$

The first equation implies that the operators $a_{m}^{\dagger}$ in the polaron Hamiltonian, Eq. (11), create a self-localized polaron because intersite hopping is negligible due to the vanishing off-diagonal $E_{m n}$ elements. The second line implies that this small polaron state is decoupled from all phonon modes as $G_{\mathbf{Q} m n}=0$. The on-site polaron energy $E_{m m}$ is the sum of the electronic energy $\varepsilon_{m m}$ of the corresponding WF and the potential energy decrease due to the lattice distortion, analogous to the CHO case [compare the second terms in Eqs. (3) and (23)]. The physical insight provided by Eq. (23) is that a material with less dispersive electronic bands, in which $\varepsilon_{m m}$ is closer to the band edge, and stronger on-site $e$-ph coupling $g_{\mathbf{Q} m m}$ (and thus greater potential energy decrease), is more likely to host a small polaron.

Whether or not a small polaron forms depends on the competition of two terms, namely the potential energy decrease due to the lattice distortion and the kinetic energy increase from localizing a Bloch state. If the on-site polaron energy $E_{m m}$ is lower than the energy of the conduction-band minimum (CBM) for an electron carrier, or higher than the valence-band maximum (VBM) for a hole carrier, then the self-localized polaron is energetically more favorable than a delocalized Bloch state. In this scenario, the electron or hole quasiparticle forms a small polaron and becomes self-trapped by the lattice distortion; the polaron formation energy is thus the difference between the polaron energy $E_{m m}$ and the respective band edge. For electron carriers, the polaron formation energy is given by

$$
\Delta E_{\mathrm{f}}^{\mathrm{el}}=E_{m m}-\varepsilon_{\mathrm{CBM}},
$$

while similarly for hole polarons the formation energy is

$$
\Delta E_{\mathrm{f}}^{\text {hole }}=\varepsilon_{\mathrm{VBM}}-E_{m m} .
$$


The small polaron wave function has rarely been discussed in the canonical transformation treatment. While Eq. (23) gives the polaron energy for an electron in a given WF, the choice of a WF is not unique-different WFs will result in slightly different lattice distortions and polaron energies, the most stable state corresponding to the WF minimizing the polaron energy. In the following, we use a maximally localized WF as a trial wave function and compute its polaron energy. If the resulting small polaron is stable, as determined by comparing the electron or hole polaron energy with the respective band edge, then our approach provides a sufficient condition for concluding that a small polaron forms in the material, as well as an approximate polaron wave function.

\section{FIRST-PRINCIPLES CALCULATIONS}

We carry out DFT calculations using the QUANTUM ESPRESSO code [44] with a plane-wave basis set, employing norm-conserving pseudopotentials [45] from Pseudo Dojo [46] and the Perdew-Burke-Ernzerhof generalized gradient approximation [47]. A kinetic energy cutoff of $100 \mathrm{Ry}$, an $8 \times$ $8 \times 8 \mathbf{k}$-point grid, and relaxed lattice parameters are used in all DFT calculations. We use density functional perturbation theory [48] to compute phonon frequencies and eigenvectors on a coarse $8 \times 8 \times 8$ q-point grid for all materials except $\mathrm{Na}_{2} \mathrm{O}_{2}$, for which we use a $4 \times 4 \times 4$ q-point grid. The $e$-ph coupling constants $g_{m n v}(\mathbf{k}, \mathbf{q})$ are obtained on coarse grids and transformed to Wannier basis coupling constants $g_{\mathbf{Q} m n}$ using the PERTURBO code [42], with WFs generated from WANNIER90 [49]. Computing the polaron formation energy with Eq. (23) only requires Wannierizing one or more bands and computing the potential energy decrease term, which has a small computational cost equal to computing an $e$-ph scattering rate [42]. Before calculating the polaron energy, we first numerically verify that the identity in Eq. (22) is satisfied. We then obtain the on-site polaron energy, $E_{m m}$ in Eq. (23), carrying out the Brillouin zone integral via Monte Carlo integration with 1 million random $\mathbf{q}$ points drawn from a Cauchy distribution. All materials investigated in this work have strongly polar bonds and dominant Fröhlich $e$-ph coupling with the longitudinal optical modes $[28,50]$. The temperature is set to $300 \mathrm{~K}$ in all calculations.

\section{RESULTS}

Figure 1 shows the computed polaron energy in three alkali halides- $\mathrm{NaCl}, \mathrm{LiF}$, and $\mathrm{KCl}-$ for both electron and hole polaron states. Our formalism predicts that holes in these three materials form a self-localized small polaron, in agreement with experiments [3], because the computed polaron energies $E_{m m}$ are above the VBM, as shown in Figs. 1(a)-1(c). Electrons in these materials, on the other hand, are not expected to self-trap-as the conduction band in alkali halides is $s$-like and therefore more dispersive than the $p$-like valence band, the potential energy decrease due to the lattice distortion cannot outweigh the increase in kinetic energy for localizing the electronic state. Consistent with this intuition, our results for electrons in $\mathrm{NaCl}, \mathrm{LiF}$, and $\mathrm{KCl}$, shown in Figs. 1(d)-1(f), conclude that electrons in these materials do not form a selftrapped polaron, as is seen by the fact that the polaron energy (a)
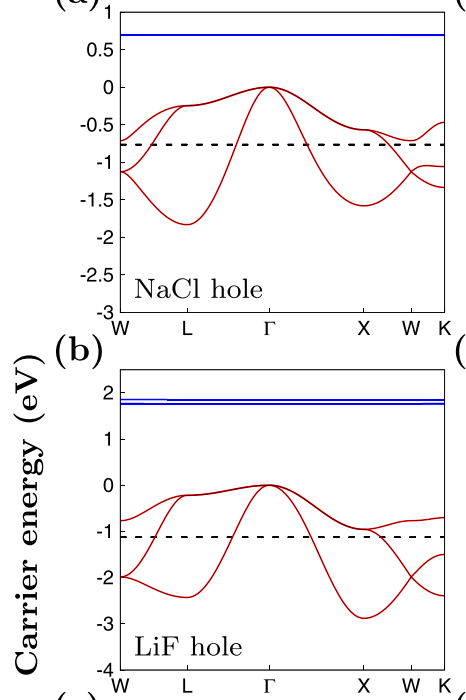

(c)

(e)
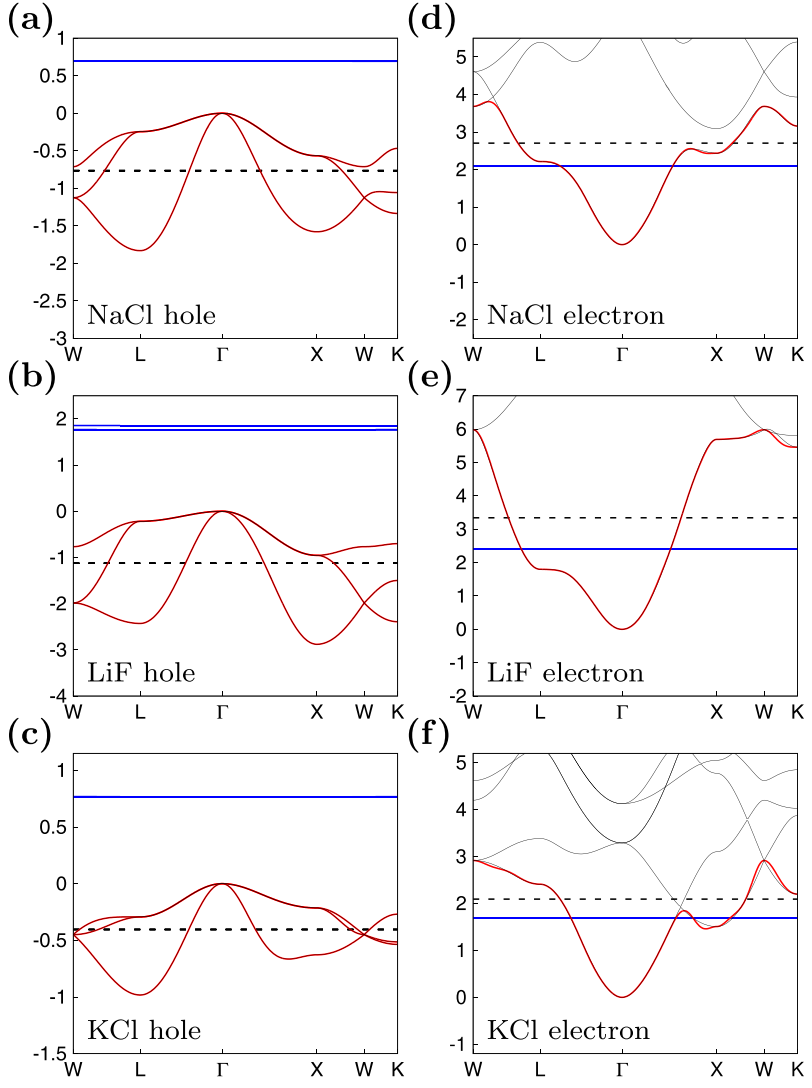

(f)

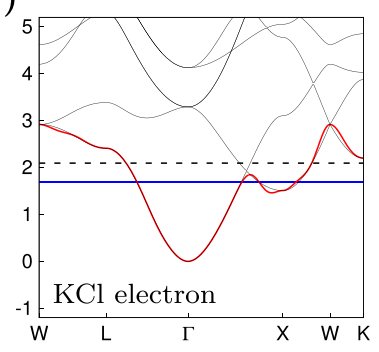

Polaron Electronic WF DFT Wannier interpolation

FIG. 1. Calculated polaron energy for holes in (a) $\mathrm{NaCl}$, (b) $\mathrm{LiF}$, and (c) $\mathrm{KCl}$, and electrons in (d) $\mathrm{NaCl}$, (e) $\mathrm{LiF}$, and (f) $\mathrm{KCl}$. Blue lines are the polaron on-site energies $E_{m m}$, and dashed black lines are WF energies $\varepsilon_{m m}$, in Eq. (23). The solid black curves are the DFT band structure, and the red curves are the Wannier interpolated bands, whose number equals the number of WFs employed in the calculation. The energy zero is set to either the CBM or VBM, respectively, when electron or hole carriers are considered.

is above the CBM. Experiments in alkali halides similarly found no evidence of electron polarons down to $5 \mathrm{~K}$ [51].

Figure 2 shows the calculated electron and hole polaron energies in three alkali-metal oxides and peroxides: $\mathrm{Na}_{2} \mathrm{O}_{2}, \mathrm{Li}_{2} \mathrm{O}_{2}$, and $\mathrm{Na}_{2} \mathrm{O}$. The nature of the charge carriers in these materials is important for application to novel battery technologies, where the low electrical conductivity hampers device performance and is commonly attributed to the presence of small polarons [9]. Our results in Fig. 2 unambiguously demonstrate that both electrons and holes in these materials form self-localized small polarons with formation energies greater than $0.5-1 \mathrm{eV}$, warranting further investigation of their electrical transport properties. The hole polaron wave function in $\mathrm{NaCl}$ and the electron polaron wave function in $\mathrm{Li}_{2} \mathrm{O}_{2}$ are shown in Figs. 3(a) and 3(b), respectively, highlighting their localized nature.

The last case study we examine is cubic $\mathrm{SrTiO}_{3}$ perovskite, whose electron mobility near room temperature exhibits a power law that can be attributed to a transport regime governed by large (non-self-localized) polarons [31]. We 
(a)

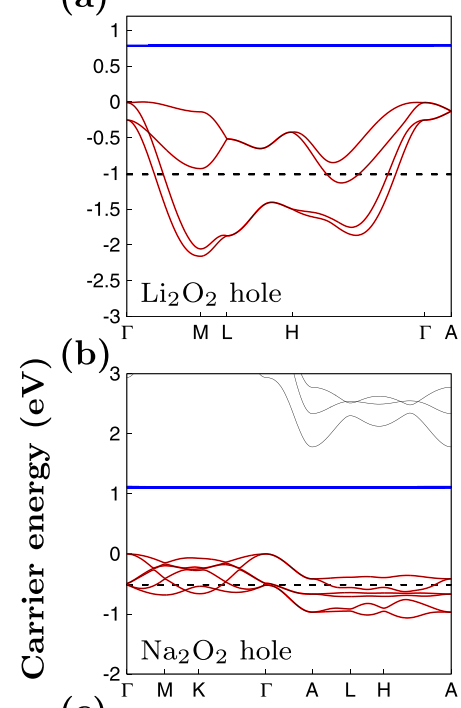

(c)

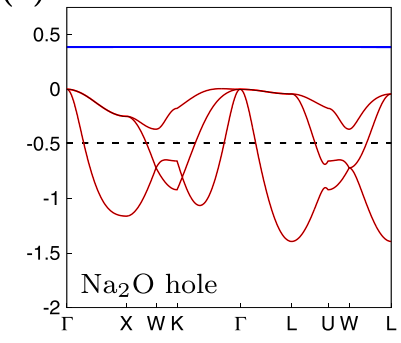

Polaron (d)

(e)
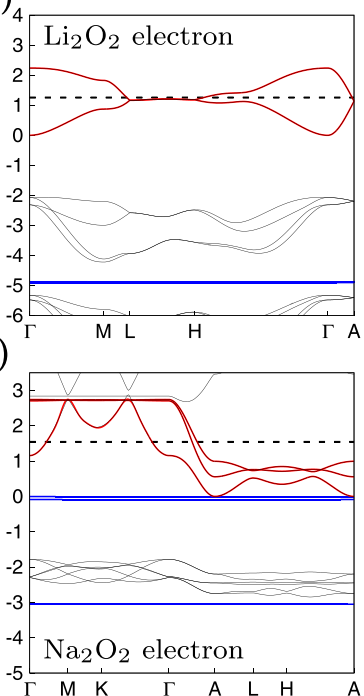

(f)

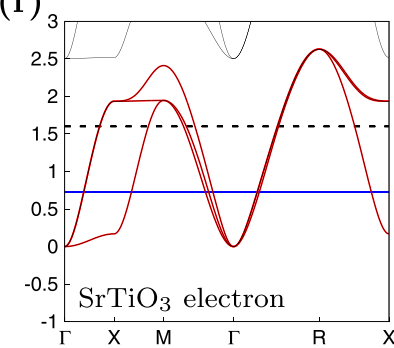

DFT

Wannier interpolation

FIG. 2. Calculated polaron energy for holes in (a) $\mathrm{Li}_{2} \mathrm{O}_{2}$, (b) $\mathrm{Na}_{2} \mathrm{O}_{2}$, and (c) $\mathrm{Na}_{2} \mathrm{O}$, and electrons in (d) $\mathrm{Li}_{2} \mathrm{O}_{2}$, (e) $\mathrm{Na}_{2} \mathrm{O}_{2}$, and (f) $\mathrm{SrTiO}_{3}$. The lines and their color code have the same meaning as in Fig. 1.

investigate small polaron formation in cubic $\mathrm{SrTiO}_{3}$, using accurate electronic band structure, phonon dispersions, and $e$-ph interactions from our previous work [30,31] as a starting point for the polaron calculation. As shown in Fig. 2(f), we find a polaron energy significantly higher than the CBM, clearly showing that for electrons in $\mathrm{SrTiO}_{3}$ it is energetically unfavorable to self-localize and form a small polaron state. Note that this finding does not conflict with the existence of localized electronic states due to oxygen vacancies [25,34] as our approach focuses on self-localized electronic states in the pristine crystal.

(a)

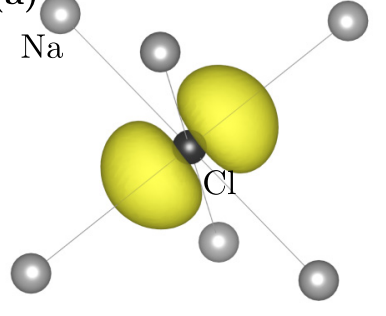

(b)

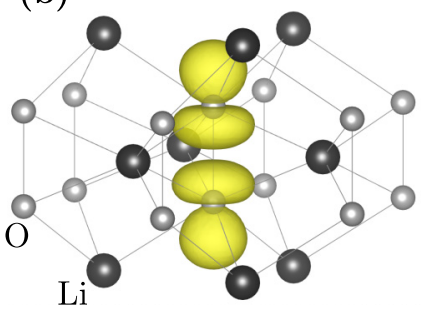

FIG. 3. The square of the trial polaron wave function for the (a) hole polaron in $\mathrm{NaCl}$ and (b) electron polaron in $\mathrm{Li}_{2} \mathrm{O}_{2}$.

\section{DISCUSSION}

The method developed in this work focuses on finding self-localized polarons with negligible intersite hopping and polaron bandwidth (and thus immobile), as is implied by Eq. (23). As shown in the case of $\mathrm{SrTiO}_{3}$, even when a stable small polaron state is not found with our approach, a more extended small polaron or a large polaron state may still be present in the system. This limitation of our approach can be overcome by removing the assumption of local $e$-ph coupling or by directly solving the polaron Hamiltonian and treating the distortion coefficients $B_{\mathbf{Q}}$ as variational parameters. Both of these possibilities lead to more challenging calculations and are left for future work. Translating recent advances in polaron models [52] into first-principles calculations may also enable accurate modeling of more delocalized polaron states. Another possibility to describe small polarons delocalized over a few unit cells would be to employ a linear combination of WFs and use a variational approach to minimize the polaron energy. Such a calculation is left for future work.

The formalism presented in this work leaves room for various straightforward extensions. One is minimizing the polaron energy over the space of possible trial WFs, leading to a refinement of the polaron formation energy and wave function. Mode-resolved analysis of the potential energy decrease is also possible, and allows one to infer which phonon modes contribute to small polaron formation. In addition, treating $e$-ph interactions in materials with open-shell $d$ or $f$ electrons, for example using the DFT $+\mathrm{U}$ approach, is an important future extension for studies of small polaron effects in transition-metal oxides [53,54]. Our approach also forms the basis for charge-transport calculations in the polaron hopping regime, for example using the Kubo formula [5,16,17], and for studies of the transition from bandlike to polaron hopping transport $[4,20,21]$. Both topics are pristine territory for first-principles calculations.

Finally, there are conceptual differences between our approach and a recently proposed momentum-space formalism to treat polarons using Landau-Pekar theory $[55,56]$. The approach proposed in Refs. [55,56] can be obtained as a special case of our treatment under simplifying assumptions. While the operator $C_{m n}$, and therefore also $e_{m n}^{-C}$, contains phonon creation and annihilation operators, let us suppose that we can take a thermal average and turn $e_{m n}^{-C}$ into a matrix, $A_{m n}$. By replacing $e_{m n}^{-C}$ with $A_{m n}$ in Eq. (13), as opposed to taking thermal averages of $\widetilde{M}_{m n}$ as we did above, the on-site energy and the pl-ph interaction in Eq. (12), for a polaron at the Bravais lattice origin $\left(\mathbf{R}_{n}=\mathbf{R}_{m}=\mathbf{0}\right)$, become

$$
\begin{aligned}
E_{00}=\sum_{m n} A_{0 m}^{*}\left[\varepsilon_{m n}-\frac{2}{N_{\Omega}} \sum_{\mathbf{Q}} \omega_{\mathbf{Q}} B_{-\mathbf{Q} 00} g_{\mathbf{Q} m n}\right] A_{n 0} \\
+\frac{1}{N_{\Omega}} \sum_{\mathbf{Q}} \omega_{\mathbf{Q}} B_{-\mathbf{Q} 00} B_{\mathbf{Q} 00}, \\
G_{\mathbf{Q} 00}=\sum_{m n} A_{0 m}^{*} g_{\mathbf{Q} m n} A_{n 0}-B_{\mathbf{Q} 00} .
\end{aligned}
$$

This result is a Wannier space version of the two polaron equations proposed in Refs. [55,56], but with an additional term (the second line of the first equation above) whose origin will be discussed elsewhere. Despite the similarities, the two 
formalisms differ in two crucial points: (i) Our approach guarantees that the polaron state is self-localized, as the polaron hopping term between nearby sites, $E_{0 n}$, is negligibly small; (ii) we use Wannier functions as a more natural, localized basis set to describe small polarons, thus bypassing costly calculations in momentum space. For example, our computed electron polaron formation energy in $\mathrm{Li}_{2} \mathrm{O}_{2}$ is $-4.905 \mathrm{eV}$ [see Fig. 2(d)], which is nearly identical to the $-4.87 \mathrm{eV}$ value extrapolated to infinite supercell sizes using progressively finer k-point grids in Ref. [55]. Remarkably, our approach obtains this value with a negligible computational cost, without the need for any extrapolation or fine k-point grid sampling, and without solving a polaron eigenvalue problem in momentum space as in Ref. [55].

\section{CONCLUSION}

In summary, we developed a computationally efficient approach to predict the formation of self-localized small polarons, and we made it available in our open-source PERTURBO code. Our formalism combines ab initio $e$-ph interactions with an extension of small polaron theory. Its computational cost is a minimal overhead to a DFT calculation on a unit cell, allowing one to rapidly scan many materials. Besides providing a convenient atomistic approach for small polaron studies, our method is a starting point for developing transport calculations in the polaron hopping regime.

\section{ACKNOWLEDGMENTS}

This work was supported by the Air Force Office of Scientific Research through the Young Investigator Program, Grant No. FA9550-18-1-0280. J.-J.Z. was supported by the Joint Center for Artificial Photosynthesis, a DOE Energy Innovation Hub, supported through the Office of Science of the U.S. Department of Energy under Award No. DE-SC0004993. H.-Y.C. acknowledges support by the J. Yang Fellowship. This research used resources of the National Energy Research Scientific Computing Center (NERSC), a U.S. Department of Energy Office of Science User Facility located at Lawrence Berkeley National Laboratory, operated under Contract No. DE-AC02-05CH11231.
[1] D. Emin, Phys. Today 35(6), 34 (1982).

[2] S. Lany, J. Phys. Condens. Matter 27, 283203 (2015).

[3] T. G. Castner and W. Känzig, J. Phys. Chem. Solids 3, 178 (1957).

[4] L. B. Schein, C. B. Duke, and A. R. McGhie, Phys. Rev. Lett. 40, 197 (1978).

[5] J. H. Fetherolf, D. Golež, and T. C. Berkelbach, Phys. Rev. X 10, 021062 (2020).

[6] H. Tuller and A. Nowick, J. Phys. Chem. Solids 38, 859 (1977).

[7] H. Sezen, H. Shang, F. Bebensee, C. Yang, M. Buchholz, A. Nefedov, S. Heissler, C. Carbogno, M. Scheffler, P. Rinke, and C. Wöll, Nat. Commun. 6, 6901 (2015).

[8] E. Pastor, J.-S. Park, L. Steier, S. Kim, M. Grätzel, J. R. Durrant, A. Walsh, and A. A. Bakulin, Nat. Commun. 10, 3962 (2019).

[9] J. Kang, Y. S. Jung, S.-H. Wei, and A. C. Dillon, Phys. Rev. B 85, 035210 (2012).

[10] S. P. Ong, Y. Mo, and G. Ceder, Phys. Rev. B 85, 081105(R) (2012).

[11] X. Wu, M. T. Trinh, D. Niesner, H. Zhu, Z. Norman, J. S. Owen, O. Yaffe, B. J. Kudisch, and X.-Y. Zhu, J. Am. Chem. Soc. 137, 2089 (2015)

[12] D. Cortecchia, J. Yin, A. Bruno, S.-Z. A. Lo, G. G. Gurzadyan, S. Mhaisalkar, J.-L. Brédas, and C. Soci, J. Mater. Chem. C 5, 2771 (2017).

[13] M. B. Salamon and M. Jaime, Rev. Mod. Phys. 73, 583 (2001).

[14] T. Holstein, Ann. Phys. (NY) 8, 343 (1959).

[15] M. I. Dykman and E. I. Rashba, Phys. Today 68(4), 10 (2015).

[16] I. G. Lang and Y. A. Firsov, J. Expt. Theor. Phys. (U.S.S.R.) 43, 1843 (1962).

[17] R. Silbey and R. W. Munn, J. Chem. Phys. 72, 2763 (1980).

[18] R. W. Munn and R. Silbey, J. Chem. Phys. 83, 1843 (1985).

[19] K. Hannewald, V. M. Stojanović, J. M. T. Schellekens, P. A. Bobbert, G. Kresse, and J. Hafner, Phys. Rev. B 69, 075211 (2004).

[20] V. N. Bogomolov, E. K. Kudinov, and Y. A. Firsov, Sov. Phys. Solid State 9, 2502 (1968) [Fiz. Tverd. Tela 9, 3175 (1967)].
[21] H. Böttger and V. V. Bryksin, Phys. Status Solidi B 78, 415 (1976).

[22] D. Emin, Phys. Rev. Lett. 28, 604 (1972).

[23] W. Meevasana, X. J. Zhou, B. Moritz, C.-C. Chen, R. H. He, S.I. Fujimori, D. H. Lu, S.-K. Mo, R. G. Moore, F. Baumberger, T. P. Devereaux, D. van der Marel, N. Nagaosa, J. Zaanen, and Z.-X. Shen, New J. Phys. 12, 023004 (2010).

[24] D. Keroack, Y. Lepine, and J. L. Brebner, J. Phys. C 17, 833 (1984).

[25] M. L. Crespillo, J. T. Graham, F. Agulló-López, Y. Zhang, and W. J. Weber, Appl. Mater. Today 12, 131 (2018).

[26] R. M. Martin, Electronic Structure: Basic Theory and Practical Methods (Cambridge University Press, Cambridge, 2004).

[27] J. J. Kas, J. J. Rehr, and L. Reining, Phys. Rev. B 90, 085112 (2014).

[28] J.-J. Zhou and M. Bernardi, Phys. Rev. B 94, 201201(R) (2016).

[29] N.-E. Lee, J.-J. Zhou, L. A. Agapito, and M. Bernardi, Phys. Rev. B 97, 115203 (2018).

[30] J.-J. Zhou, O. Hellman, and M. Bernardi, Phys. Rev. Lett. 121, 226603 (2018).

[31] J.-J. Zhou and M. Bernardi, Phys. Rev. Research 1, 033138 (2019).

[32] Y. Kang, H. Peelaers, and C. G. Van de Walle, Phys. Rev. B 100, 121113(R) (2019).

[33] N.-E. Lee, J.-J. Zhou, H.-Y. Chen, and M. Bernardi, Nat. Commun. 11, 1607 (2020).

[34] A. Janotti, J. B. Varley, M. Choi, and C. G. Van de Walle, Phys. Rev. B 90, 085202 (2014).

[35] S. Kokott, S. V. Levchenko, P. Rinke, and M. Scheffler, New J. Phys. 20, 033023 (2018).

[36] S. Yuan, Z. Wang, M. L. F. Baron, and K. H. Bevan, Phys. Rev. B 100, 205201 (2019).

[37] N. Tsunoda, Y. Kumagai, and F. Oba, Phys. Rev. Materials 3, 114602 (2019).

[38] J. T. Devreese, Encycl. Appl. Phys. 14, 383 (1996).

[39] Note that here we define a small polaron as a self-trapped electronic state, regardless of its spatial extent. 
[40] G. D. Mahan, Many-Particle Physics, 3rd ed. (Springer, New York, 2000).

[41] N. Marzari, A. A. Mostofi, J. R. Yates, I. Souza, and D. Vanderbilt, Rev. Mod. Phys. 84, 1419 (2012).

[42] J.-J. Zhou, J. Park, I.-T. Lu, X. Tong, and M. Bernardi, Comput. Phys. Commun. 264, 107970 (2021).

[43] In deriving the polaron Hamiltonian, we assumed that the carrier concentration is low enough that polaron-polaron interactions can be neglected.

[44] P. Giannozzi, S. Baroni, N. Bonini, M. Calandra, R. Car, C. Cavazzoni, D. Ceresoli, G. L. Chiarotti, M. Cococcioni, I. Dabo, A. D. Corso, S. de Gironcoli, S. Fabris, G. Fratesi, R. Gebauer, U. Gerstmann, C. Gougoussis, A. Kokalj, M. Lazzeri, L. Martin-Samos et al., J. Phys.: Condens. Matter 21, 395502 (2009).

[45] N. Troullier and J. L. Martins, Phys. Rev. B 43, 1993 (1991).

[46] M. van Setten, M. Giantomassi, E. Bousquet, M. Verstraete, D. Hamann, X. Gonze, and G.-M. Rignanese, Comput. Phys. Commun. 226, 39 (2018).
[47] J. P. Perdew, K. Burke, and M. Ernzerhof, Phys. Rev. Lett. 77, 3865 (1996).

[48] S. Baroni, S. de Gironcoli, A. Dal Corso, and P. Giannozzi, Rev. Mod. Phys. 73, 515 (2001).

[49] A. A. Mostofi, J. R. Yates, G. Pizzi, Y.-S. Lee, I. Souza, D. Vanderbilt, and N. Marzari, Comput. Phys. Commun. 185, 2309 (2014).

[50] V. A. Jhalani, J.-J. Zhou, J. Park, C. E. Dreyer, and M. Bernardi, Phys. Rev. Lett. 125, 136602 (2020).

[51] W. H. Duerig, Phys. Rev. 86, 565 (1952).

[52] Y.-C. Yam, M. M. Moeller, G. A. Sawatzky, and M. Berciu, Phys. Rev. B 102, 235145 (2020).

[53] M. Setvin, C. Franchini, X. Hao, M. Schmid, A. Janotti, M. Kaltak, C. G. Van de Walle, G. Kresse, and U. Diebold, Phys. Rev. Lett. 113, 086402 (2014).

[54] F. Freytag, G. Corradi, and M. Imlau, Sci. Rep. 6, 36929 (2016).

[55] W. H. Sio, C. Verdi, S. Poncé, and F. Giustino, Phys. Rev. Lett. 122, 246403 (2019).

[56] W. H. Sio, C. Verdi, S. Poncé, and F. Giustino, Phys. Rev. B 99, 235139 (2019). 